\title{
Uso agrícola del suelo y demanda de agua para riego en la cuenca del río Vinces (Ecuador) durante el período 1990 - 2014
}

\author{
Agricultural land use and water demand for irrigation in the Vinces basin (Ecuador), period \\ $1990-2014$
}

\author{
Historial del artículo \\ Recibido: \\ 05 de abril de 2020 \\ Revisado \\ 26 de mayo de 2020 \\ Aceptado: \\ 19 de junio de 2020
}

\section{José Luis Muñoz Marcillo ${ }^{\mathrm{a}}$, Jorge Gentilib, Roberto Bustos Cara ${ }^{\mathrm{c}}$}

\section{Palabras clave}

Cambio temporo-espacial, cuenca río Guayas, productos agrícolas, SIG, uso del suelo

\section{Keywords}

Agricultural products, GIS, Guayas river basin, land use, temporo-spatial change

\author{
a Universidad Técnica Estatal de Quevedo, Quevedo, Ecuador. Correo electrónico: jsmunoz@uteq.edu.ec \\ b Universidad Nacional del Sur-CONICET, Bahía Blanca, Argentina. \\ c Universidad Nacional del Sur, Bahía Blanca, Argentina.
}

\begin{abstract}
Resumen
El rápido crecimiento demográfico y la creciente demanda internacional de productos agrícolas tropicales han propiciado la conversión de extensas áreas de tierra para la producción agrícola intensiva de varios monocultivos en el Ecuador. El presente trabajo tiene por objetivo analizar el cambio temporo-espacial del uso del suelo agrícola y la ampliación de su frontera en la cuenca del río Vinces durante el período 1990-2014 por efecto de la presencia de los principales monocultivos agrícolas y la demanda de agua para riego por parte de los mismos cultivos agrícolas, de acuerdo a las concesiones de agua otorgadas por la autoridad gubernamental con competencia en la cuenca. La presente investigación conllevó la recopilación de geoinformación digital, su posterior procesamiento en Sistemas de Información Geográfica, tabulación de información estadística oficial y verificación en terreno. La integración conceptual de la Gestión Integrada de Recursos Hídricos permite avanzar en la comprensión de la complejidad y las dificultades para alcanzar un equilibrio entre los procesos naturales y sociales que nos afectan. Las coberturas agrícolas principales de la cuenca del río Vinces representada por los monocultivos de banano, cacao, y palma aceitera presentaron un notable y progresivo cambio temporo-espacial (ampliación de la superficie cultivada) y aumento de la presión por el agua de la cuenca para el riego en el largo período de estiaje. Las concesiones históricas de agua para riego extendidas en la cuenca del río Vinces por parte de la autoridad ambiental no refleja el verdadero volumen que emplea el sector agrícola en la etapa de verano, lo cual queda demostrado al relacionar los volúmenes de agua concesionados, las hectáreas de cultivos plantadas y los requerimientos reales de agua por los cultivos antes mencionados para lograr su máxima productividad.
\end{abstract}

\section{Abstract}

The rapid population growth and the growing international demand for tropical agricultural products have led to the conversion of large areas of land for the intensive agricultural production of several monocultures in Ecuador. The aim of the present work is to analyze the temporo-spatial change of agricultural land use and the expansion of its border in the Vinces basin during the period 1990-2014 due to the presence of the main agricultural monocultures and the demand for water for irrigation by the same agricultural crops according to the water concessions granted by the governmental authority with competence in the basin. The present investigation involved the compilation of digital geoinformation, its subsequent processing in Geographic Information Systems, tabulation of official statistical information and verification in the field. The conceptual integration of the Integrated Management of Water Resources allows us to advance in the understanding of the complexity and difficulties to reach a balance between the natural and social processes that affect us. The main agricultural coverings of the Vinces river basin, represented by monocultures of banana, cocoa, and oil palm, showed a remarkable and progressive temporo-spatial change (expansion of the cultivated area) and an increase in water pressure in the basin. irrigation in the long period of low water. The historical concessions of irrigation water extended in the Vinces basin by the environmental authority does not reflect the true volume used by the agricultural sector in the summer stage, which is demonstrated by relating the volumes of water concessioned, the hectares of planted crops and the actual water requirements for the crops mentioned above to achieve maximum productivity. 


\section{Introducción}

El cambio de usos del suelo ha sido abordado desde distintos enfoques, con múltiples finalidades: instrumentos de control fiscal, evaluación ambiental, entre otros. Es en este último aspecto donde adquiere gran relevancia, ya que los cambios en la cobertura del suelo que incluyen altas tasas de deforestación se consideran como los principales inductores a la degradación ambiental, fragmentación de la tierra y pérdida de biodiversidad a escala global (Lambin \& Turner, 2001). También adquiere relevancia el análisis de los usos del suelo, su variación en el tiempo y en el espacio, y su respectiva demanda de riego en relación con la Gestión Integrada de los Recursos Hídricos.

La ciencia del cambio de cobertura de la tierra, el cambio de su uso y el análisis geográfico de las mismas utilizan conceptos y métodos a partir de la Ecología Humana y Cultural, las dimensiones humanas del cambio global, la ciencia regional, la ciencia de los SIG, la teledetección y la ecología del paisaje (Zimmerer, 2010). Según Rindfuss et al. (2007), el enfoque amplio de conceptos y métodos que se utiliza en el análisis del cambio del uso de la tierra se combina con las interacciones económicas y socioeconómicas de la toma de decisiones con base en el análisis espacial apoyado en la evaluación del cambio de la cobertura de la tierra mediante imágenes de sensores remotos. En este sentido, se concibe a la teledetección como una herramienta muy adecuada para el mapeo de grandes superficies de terreno, lo que resulta rentable para el estudio de áreas agrícolas. Sin embargo, el monitoreo basado en la teledetección para la agricultura en áreas extensivas de pequeña escala donde se requiere datos con alta resolución espacial y temporal suelen ser insuficientes para una delimitación precisa (Knauer, Gessner, Fensholt, Forkuor, \& Kuenzer, 2017).

La magnitud del impacto humano sobre nuestro medio ambiente y la necesidad de predecir cambios futuros frente a las crecientes poblaciones humanas exigen un papel fundamental de la Geografía Física que a través de un nuevo enfoque geográfico apoyado en subdisciplinas como biogeografía, climatología, geomorfología, hidrología e interacción humano-ambiente permitan el análisis de los problemas ambientales dentro del contexto del desarrollo sostenible a escalas local, nacional y global (Gregory, Gurnell \& Petts, 2002).

El rápido crecimiento demográfico y la creciente demanda internacional de productos tropicales han propiciado la conversión de extensas áreas de tierra para la producción agrícola intensiva de varios monocultivos en el Ecuador.
Para Gudynas (2013), la expansión de los monocultivos en regiones del mundo implica una explotación de los recursos naturales en grandes volúmenes o alta intensidad, orientada esencialmente a la exportación de materias primas sin procesar o con un procesamiento limitado. La expansión del monocultivo para exportación en los países de Latinoamérica constituye un ejemplo de las "actividades extractivas" o "extractivismo" que impulsó el neoliberalismo a partir de la década de los noventa (Gudynas, 2013; Seoane, 2013). De esta manera, las corporaciones transnacionales recibieron múltiples incentivos por parte del Estado cobijadas en el supuesto de que constituyen la vía más rápida al progreso económico (Svampa, 2008). En la práctica se dio paso a la mercantilización y apropiación privada de los bienes naturales en territorios con una legislación ambiental deficitaria (Silvetti, Soto, Cáceres \& Cabrol, 2013). El extractivismo en la agroexportación se muestra como una práctica en la cual se pueden encontrar los actores clave para su impulso: las empresas que se benefician, así como los conflictos y los daños que causan (Yacoub, Duarte \& Boelens, 2015).

La evolución de los cultivos de agroexportación presenta valores crecientes entre los países de América. En Argentina en los períodos comprendidos entre 1990-1991 y 2011-2012, de los 14,4 millones de hectáreas que se incorporaron a la producción a nivel nacional, el $95 \%$ fueron dedicadas al cultivo de soja (Zeolla, 2013). La producción de soja pasó con una superficie implantada de 5,9 millones de hectáreas a una superficie implantada de 19,7 millones de hectáreas. En Costa Rica, el área cultivada de piña pasó de 12.500 hectáreas en el 2000 a 42.000 hectáreas en el 2012 (SEPSA, 2001; SEPSA, 2013). Sin embargo, los cálculos de varias organizaciones ambientales dan cuenta que las hectáreas dedicadas a la piña son muy mayores a las cifras oficiales y la expansión continúa sin una planificación adecuada por parte del Estado (Chacón, 2012).

En México, como consecuencia del crecimiento de la frontera agropecuaria en la localidad de Jalisco, la cobertura vegetal del suelo correspondiente a bosque tropical ha sido la más afectada en cuarenta y tres años reduciéndose en un $17 \%$, pese a la existencia de instrumentos de planeación como los ordenamientos ecológicos y los planes de manejo de cuencas hidrográficas (Nené-Preciado, González, Mendoza \& Silva, 2017). La deforestación general que ocurre en los trópicos es una consecuencia de los cambios que sufre la cubierta terrestre a escalas local y regional como consecuencia de factores socioeconómicos, demográficos y biofísicos lo cual explican los patrones espaciales de uso de la tierra en este ecosistema (Lambin \& Mayfroidt, 2011; Pan et al., 2004). La diversificación agrícola es importante 
para los sectores rurales de los países en desarrollo, pero requiere grandes inversiones en infraestructura, capital humano bien capacitado, inversión en investigación y trabajos de extensión para difundir la diversificación en la región (Goletti, 1999).

El conocimiento de los factores inmersos dentro de la adecuada diversificación de las tierras agrícolas es una base muy importante sobre la cual los gobiernos pueden implementar políticas y proyectos para mejorar el sector de la agricultura, de manera de reducir la pobreza, particularmente para las personas que viven en el sector rural (Damiani, 2001). En el Objetivo 14 de la Agenda 21 sobre desarrollo rural y agricultura, la Comisión Brundtland subraya que los gobiernos locales deberían promover modelos de producción agrícola diversificados para apoyar una agricultura sostenible y respetuosa con el medio ambiente (Keating, 1993). La cobertura forestal ampliada en las llamadas nuevas transiciones forestales secundarias se ha vuelto cada vez una práctica más común (Ramankutty, Heller \& Rhemtulla, 2010).

En Ecuador, la producción agrícola en las décadas de 1920 y 1930 fue dominada por el cacao y a partir de la década de 1950, hasta la actualidad, el banano ha sido el producto de exportación agrícola más importante. La superficie dedicada a estos cultivos de exportación ha ido en aumento, de manera que entre 1980 y 2000 el área de cosecha se ha incrementado, llegando a 165.000 hectáreas en banano y 433.00 hectáreas para el cacao (MAGAP, 2012). La diversificación agrícola como base del cambio en el proceso productivo a nivel de provincia es muy compleja de llevar a cabo, debido principalmente a que se utiliza exhaustivamente factores de producción como mano de obra, tierra y capital y tecnología agrícola básica (Pacheco, Ochoa-Moreno, Ordoñez \& IzquierdoMontoya, 2018).

En Ecuador, como en varios países de Centroamérica y Sudamérica, se ha concentrado y acumulado agua por medio de los cultivos agrícolas comerciales de agroexportación, como es el caso del cultivo de plátano y palma de aceite, en cuyo entorno gira un entramado de medios institucionales, infraestructura hidroagrícola, paquetes de fertilizantes y subsidios gubernamentales para que las grandes empresas de alimentos concentren y posean agua, y tierra (Yacoub, Duarte \& Boelens, 2015). De esta manera, las empresas trasnacionales restringen las posibilidades de los pequeños agricultores de acceder al agua en condiciones de equidad y calidad para el riego de sus parcelas. Una preocupación general en la gestión del agua de riego en el Ecuador es reducir la presión sobre los recursos hídricos locales, lo cual se puede lograr al aumentar la eficiencia de los sistemas de riego, como se presenta en el Plan Nacional de Riego en Ecuador que exige tecnología de riego y eficiencia del sistema. La sobrextracción de agua se puede reducir con sistemas de riego modernizados, lo que ayuda a redistribuir el agua entre sectores como la agricultura, hogares y ecosistemas, aunque a nivel de captación de agua de consumo para riego neto no se realizan ahorros. Incluso un aumento en el consumo de agua puede ocurrir con una mayor eficiencia de los sistemas de riego (Salmoral, Khatun, Llive \& Madrid, 2018).

La población en Ecuador ha crecido de 4,5 millones de habitantes en 1960 a 14,9 millones de habitantes en 2010 (World Bank, 2018), como consecuencia se han intensificado las actividades antropogénicas, tales como la construcción urbana, la industria, la agricultura, la acuicultura y la deforestación (Damanik-Ambarita et al., 2018). El pastoreo intensivo y continuo, una práctica común en la cuenca del río Guayas, ha mostrado impactos negativos en la calidad del agua, lo que reduce la vegetación ribereña, modifica los canales y arroyos, aumenta la escorrentía y la erosión, así como la entrada de sedimentos (Raymond \& Vondracek, 2011).

La cuenca del río Guayas es la mayor cuenca hidrográfica de la costa del Pacífico de América del Sur, se encuentra dividida en siete subcuencas, de las que destaca por su amplio gradiente altitudinal y alta productividad agrícola la subcuenca del río Vinces. Dicha cuenca ocupa en el norte parte de las provincias de Santo Domingo de los Tsáchilas y Cotopaxi de la región sierra, y parte de la provincia de Los Ríos en la región costa. La cuenca del río Guayas está conformada por la confluencia de sus ríos principales, el Daule y Babahoyo, drenando una superficie total de $34.000 \mathrm{~km}^{2}$ distribuidas entre diversos ecosistemas que incluyen zonas de manglares, bosques secos y húmedos, páramo andino y territorio usado para actividades agrícolas. La influencia de la cuenca sobre el territorio ecuatoriano es muy importante: en 2010 la cuenca del río Guayas cubría 380.840 hectáreas de tierra irrigada, lo que es el $57 \%$ del área irrigada agrícola del Ecuador, de acuerdo a lo definido por Changjiang Institute of Survey, Planning, Design and Research (CISPDR, 2014; CISPDR, 2015).

La cuenca se encuentra bajo un clima tropical húmedo que comprende una temporada de lluvias de diciembre a mayo y una temporada seca para los meses restantes. Hay variaciones de precipitación desde el norte (2.900 a $3.100 \mathrm{~mm}$ ) hacia el sur (300 a $700 \mathrm{~mm}$ ) (CISPDR, 2015), la cuenca del Guayas dentro de la producción 
agrícola nacional incluye los cultivos más importantes de la región como el arroz (96\%), banano (68\%), caña de azúcar (97\%), maíz (55\%), café (33\%) y aceite de palma (19\%) (MAGAP, 2015). Las tierras agrícolas cubren el 49 $\%$ de la cuenca del río Guayas, seguida por los bosques (29\%) y los pastos (13\%) (Frappart et al., 2017). El río Guayas presenta una descarga anual de 30 mil millones de $\mathrm{m}^{3}$ de agua, lo que pondría a disposición $8.847 \mathrm{~m}^{3} /$ $\mathrm{hb} / \mathrm{año}$, superior a la media mundial de $6.783 \mathrm{~m}^{3} / \mathrm{hb} / \mathrm{año}$ (INOCAR, 2010).

Cuando el suelo en la cuenca del río Vinces se convierte de secano a condiciones de riego, el rendimiento del cultivo aumentará debido a una aplicación constante de agua durante todo el desarrollo de este. Espinosa \& Rivera (2016) indican que no seguir un equilibrio adecuado del agua del suelo y aplicar una dosis de riego adecuada en un momento dado puede provocar escasez o exceso de riego. Al final, los cronogramas de riego de cultivos apropiados pueden ayudar a los agricultores a comprender los requisitos de agua de los cultivos e irrigar en consecuencia. Hasta la fecha, en Ecuador todavía existe una gestión inadecuada del agua para el riego. Los agricultores de la cuenca del río Vinces producen en gran medida para el consumo nacional y las exportaciones, como el caso del arroz y plátano que son monocultivos que reúnen asociaciones de productores agrícolas que les dan un mayor acceso a los mercados nacionales e internacionales (Gaybor, Ramos, Tamayo \& Arroyo, 2008).

El presente trabajo tiene por objetivo analizar el cambio temporo-espacial del uso del suelo agrícola y la ampliación de su frontera en la cuenca del río Vinces durante el período 1990-2014 por efecto de la presencia de los principales monocultivos agrícolas de la zona y la demanda de agua para riego por parte de los mismos cultivos agrícolas. La importancia de este análisis está dada en las evidencias más importantes sobre los efectos del cambio climático para Ecuador. La dinámica del sistema climático ecuatoriano responde a la interacción de varios factores, principalmente las corrientes marinas cálida y fría del océano Pacífico. Dichas corrientes en el presente y a futuro pueden presentar potenciales alteraciones que podrían incrementar la vulnerabilidad de las zonas con déficit hídrico, como la provincia costera o los valles centrales andinos (Cadilhac, Torres, Calles, Vanacker \& Calderón, 2017). Asimismo, si se tiene en cuenta que más del $68 \%$ de la producción de los cultivos se originan en áreas irrigadas de tierras bajas en la costa central ecuatoriana (Borbor- Cordova, Boyer, McDowell \& Hall, 2006), un déficit importante de agua que no garantice el efectivo riego de amplias áreas agrícolas ocupadas por monocultivos de agroexportación cuya alta tasa de producción se concentra en los meses de verano equivalentes a las dos terceras partes del año donde la pluviometría es mínima. Sumado a esto, es de interés considerar que los proyectos de riego existentes en la cuenca del río Vinces no abastecen la demanda agrícola $\mathrm{y}$ carecen de funcionalidad por un escaso mantenimiento y control en los proyectos establecidos..

\section{Materiales y Métodos}

\section{Área de estudio}

La cuenca del río Vinces es una subcuenca de la cuenca del río Guayas que se extiende entre los paralelos $00^{\circ}$ $14^{\prime} \mathrm{S}, 02^{\circ} 27^{\prime} \mathrm{S}$ y los meridianos $78^{\circ} 36^{\prime} \mathrm{O}, 80^{\circ} 36^{\prime} \mathrm{O}$, abarca los territorios parciales o totales de ocho de las veinte y cuatro provincias ecuatorianas: Guayas, Los Ríos, Manabí, Santo Domingo de los Tsáchilas, Cotopaxi, Bolívar, Chimborazo y Cañar. A su vez, las provincias de Guayas y de Los Ríos representan juntas el 48\% de la superficie de la cuenca y el $72 \%$ de su población. El área total cubre aproximadamente $32.219 \mathrm{~km}^{2}$ (Fig. 1). Además de la cuenca del río Vinces, la cuenca del río Guayas se compone de otras seis subcuencas (SENAGUA, 2009), las cuales son de norte a sur las subcuencas de los ríos Daule, Macul, Babahoyo, drenajes menores, Juján y Yaguachi (figura 1).

La cuenca del río Vinces se ubica desde el sector nororiental al centro de la cuenca del río Guayas, extendiéndose por 426.800 hectáreas y recorre $267,96 \mathrm{~km}$ de distancia en su eje hídrico principal, sigue un sentido norte-sur constituyéndose en parte importante de la cuenca del río Guayas. Abarca el 57\% de la superficie de la provincia de Los Ríos, territorio de la costa ecuatoriana eminentemente agrícola con presencia de cultivos tropicales y subtropicales de exportación como abacá, arroz, banano, café, cacao, maíz, palma aceitera, entre otros. La cuenca del río Vinces toma su nombre del río Vinces que la atraviesa en sentido norte- sur. Este río adquiere diferentes nombres a lo largo de su recorrido, de tal manera que, en la parte norte, entre los cantones Santo Domingo de Los Tsáchilas y San Jacinto de Buena Fe, se denomina río Baba y de allí hacia el sur de la cuenca toma el nombre del cantón por donde atraviesa, así tenemos que se denomina, río Quevedo, río Mocache y río Vinces. El río Quevedo se forma de la convergencia de tres ríos los cuales son los ríos Baba, Lulo y San Pablo (figura 1).

La determinación del cambio de la cobertura agrícola de la cuenca del río Vinces se realizó a partir de la compilación de estudios de coberturas de uso del suelo generados 


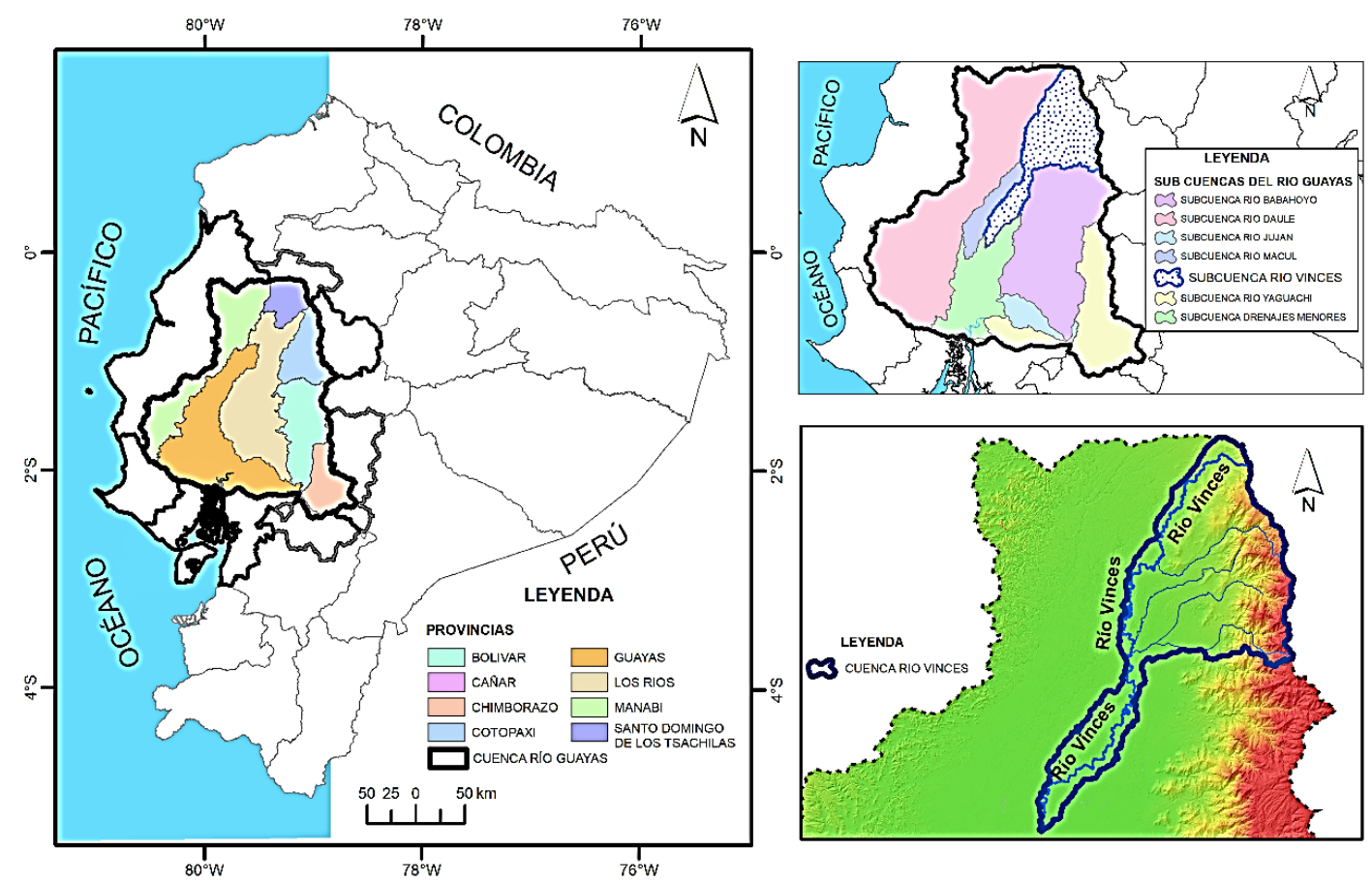

Figura 1. Área de estudio del uso agrícola del suelo y demanda de agua para riego en la cuenca del río Vinces (Ecuador). Fuente: Elaboración propia.

por el Ministerio de Agricultura y Ganadería (MAG) y Ministerio de Agricultura, Ganadería, Acuacultura y Pesca (MAGAP) para los años 1990, 2002 y 2014, lo que se complementó con el procesamiento digital de imágenes satelitales. Para cada una de las fechas analizadas se realizó un procesamiento diferencial en el entorno de Sistemas de Información Geográfica (SIG) mediante el software ArcGIS 9.3:

- Año 1990: se tomó como base la cobertura analógica del suelo realizada por MAGAP, la cual fue sometida a un proceso de digitalización y conversión al Datum vigente oficial en el Ecuador, WGS84, posteriormente se la mejoró realizando una sobreposición con imágenes para el año 1990 con el programa Google Earth Pro y una clasificación supervisada empleando una imagen del satélite Landsat 5.

- Año 2002: se tomó como base la cobertura analógica del suelo realizada por el MAGAP, la cual fue sometida a un proceso de digitalización y conversión al Datum vigente oficial en el Ecuador, WGS84, posteriormente se la mejoró realizando una sobreposición con imágenes para el año 2002 con el programa Google Earth Pro y una clasificación supervisada empleando una imagen del satélite Landsat 7.

- Año 2014: se tomó como base la cobertura digital del suelo realizada por el MAGAP - SENPLADES, la cual fue complementada con información cuantitativa de los censos de banano y cacao realizados por el MAGAP en el año 2013 y con verificación en terreno de los cultivos de banano, cacao y palma aceitera.

La determinación de la demanda de agua para riego en la cuenca del río Vinces se realizó partiendo del análisis de las concesiones otorgadas a los usuarios de la cuenca por parte de la Secretaría del Agua (SENAGUA) en las últimas décadas. Se tomaron también datos del Plan Hidráulico Regional de la Demarcación Hidrográfica Guayas (CISPDR, 2016) en lo correspondiente a la oferta y demanda hídrica para riego agrícola. Estos datos fueron analizados en forma conjunta con la superficie de monocultivos agrícolas intensivos existentes en la cuenca del río Vinces (MAGAP, 2014). Se consideraron los requerimientos de riego por ha/año en la época de verano del banano, la palma aceitera y el cacao y se realizaron comprobaciones amplias en campo. 


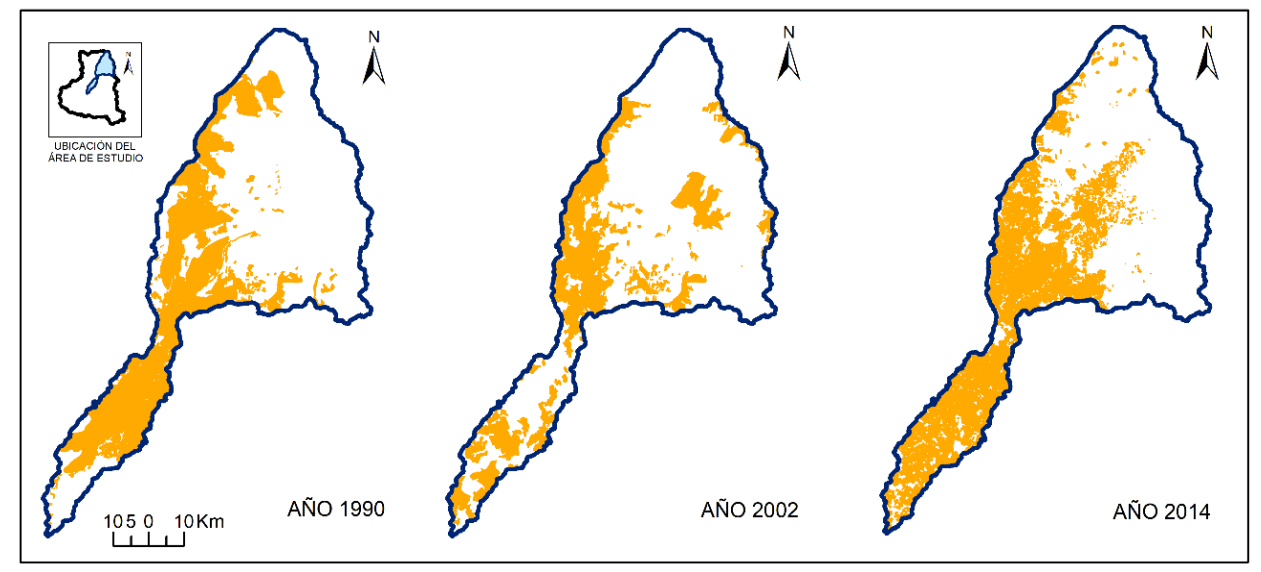

Figura 2. Cobertura agrícola en la cuenca del río Vinces (Ecuador) para los años 1990, 2002 y 2014. Fuente: Elaboración propia en base a IGM - MAGAP.

\section{Resultados}

\section{Uso del suelo}

El análisis de las coberturas agrícolas de la cuenca del río Vinces para los años 1990, 2002 y 2014 muestra un aumento en la cobertura agrícola como consecuencia de la ampliación de la frontera agrícola a partir del establecimiento de varios monocultivos como banano, cacao y palma y de cultivos intensivos de ciclo corto como el maíz, arroz, etc. (figura 2 y Tabla 1).

De la cobertura agrícola de la cuenca del río Vinces se destacan los monocultivos permanentes e intensivos de banano, cacao y palma aceitera por requerir riego continuo y abundante durante los ocho meses que dura la etapa de verano en esta región, sumado al hecho que es precisamente en la época de estiaje donde estos cultivos exóticos presentan los mayores rendimientos productivos. La necesidad de riego en promedio para la producción de banano en haciendas bananeras de la provincia de El Oro es de $27.500 \mathrm{~m} 3$ de agua para riego por ha/año de cultivo (Zarate \& Kuiper, 2013). Por otra parte, los cultivos de cacao y palma aceitera que cuentan con una estructura vegetal leñosa, de acuerdo a lo manifestado en comunicación oral por informantes clave del sector, demandarían aproximadamente la mitad del agua que consume una hectárea de banano en producción. La distribución espacial del banano, el cacao y la palma africana en la cuenca del río Vinces se presentan en la figura 3. Para el caso del banano, uno de los principales cultivos de exportación del Ecuador, en el período presentado
Tabla 1

Uso agrícola en 1990, 2002 y 2014 en la cuenca del río Vinces (Ecuador).

\begin{tabular}{llll} 
Cobertura del suelo & $\begin{array}{l}\text { año 1990 } \\
\text { MAG } \\
\text { Área (ha) }\end{array}$ & $\begin{array}{l}\text { año 2002 } \\
\text { MAGAP } \\
\text { Área (ha) }\end{array}$ & $\begin{array}{l}\text { año 2014 } \\
\text { MAGAP } \\
\text { Área (ha) }\end{array}$ \\
\hline $\begin{array}{l}\text { Arboricultura Tropical } \\
\text { Cultivos de Ciclo Corto }\end{array}$ & $11.280,55$ & $24.843,77$ & $23.932,09$ \\
Cultivos de Banano & $56.586,61$ & \\
Cultivos de Palma Africana & $21.399,05$ & $15.166,85$ & $20.543,46$ \\
Cultivos de Maíz & $11.401,8$ & $36.87,91$ & $40.484,22$ \\
Cultivos de Café & $35.141,7$ & $34.13,40$ & \\
Cultivos de Arroz & 5.760 & $14.48,62$ & $3.444,17$ \\
Cultivos de Caña de Azúcar & $2.219,96$ & $10.02,33$ & 27,82 \\
Cultivos de Cacao & $10.983,95$ & 841,89 & $36.129,02$ \\
Cultivo de Café-Cacao & $49.332,79$ & & \\
Abacá & & & 595,67 \\
Chía & & & 58,13 \\
Palmito & & & 762,61 \\
Tabaco & & & $1.368,51$ \\
\hline Total & $147.519,80$ & $106.991,37$ & $127.345,70$ \\
\hline
\end{tabular}

Fuente: Elaboración . propia en base a IGM - MAGAP.

muestra una expansión del 112\%. (figura 3a). En el caso del monocultivo de cacao muestra una expansión del $800 \%$ (figura 3b). Por otro lado, el monocultivo de palma aceitera o palma africana presenta una ligera disminución del 0,04\% (figura 3c). 

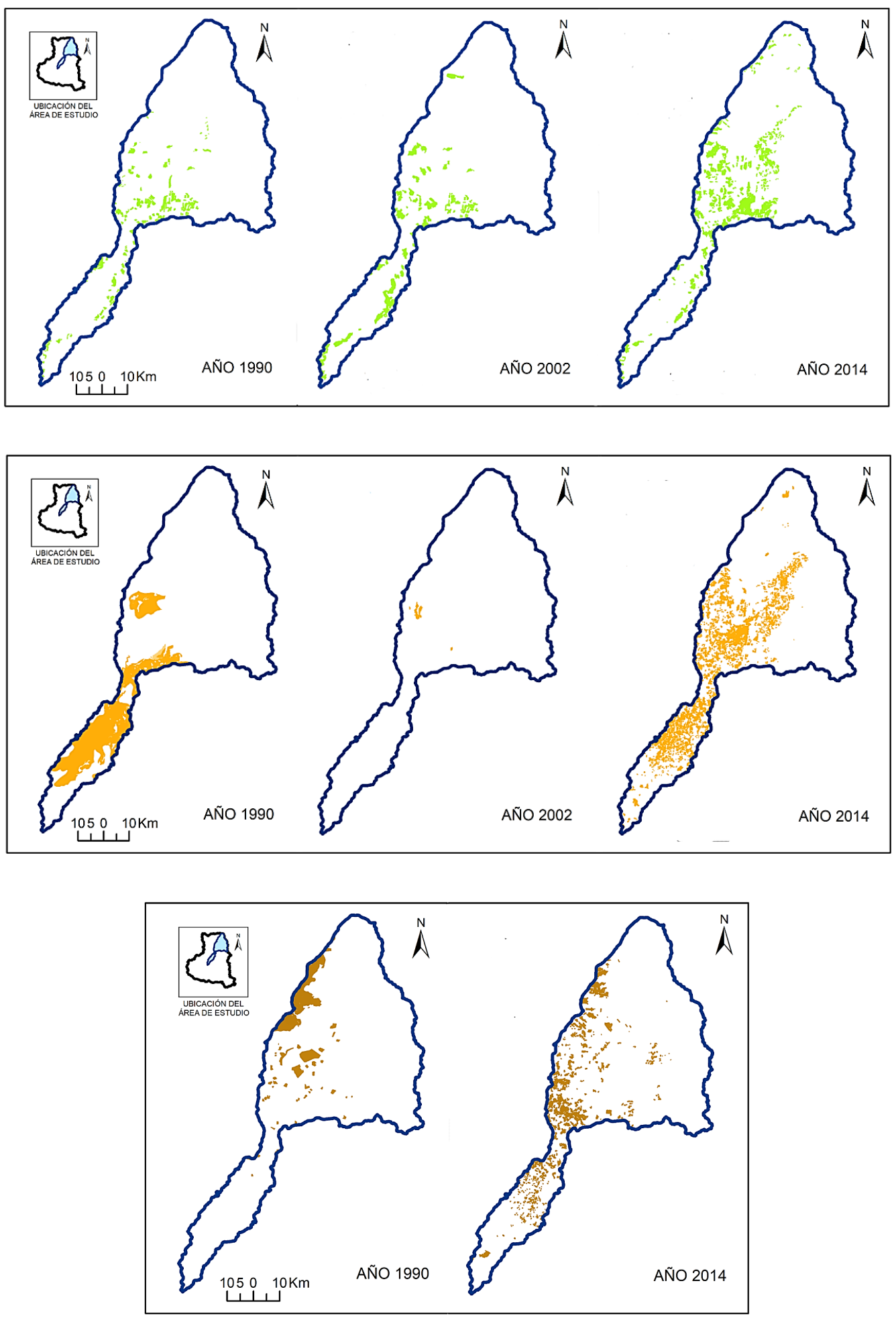

Figura 3. Evolución de la cobertura de a) banano b) cacao y c) palma africana en la cuenca del río Vinces (Ecuador). Fuente: Elaboración propia en base a IGM - MAGAP. 


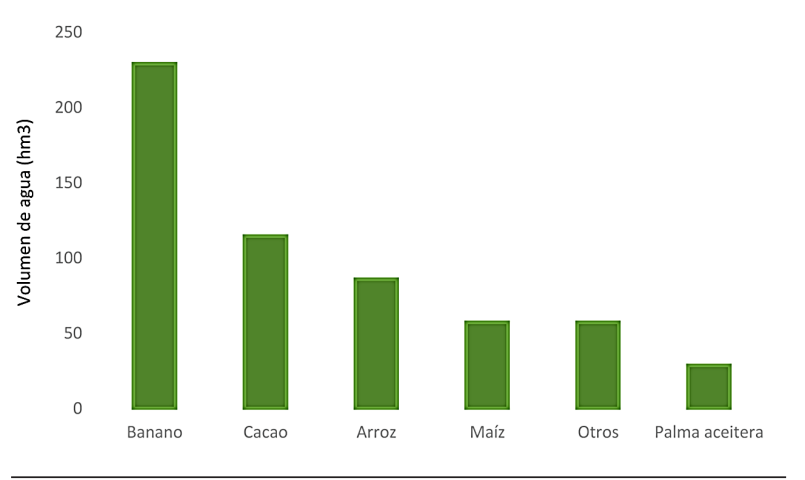

Figura 4. Distribución de $573,06 \mathrm{Hm} 3$ agua para riego de cultivos agrícolas en cuenca del río Vinces (Ecuador). Fuente: Elaboración propia.

El cultivo de banano en el año 1990 ocupaba mayormente la parte central de la cuenca del río Vinces, mientras que para el año 2002 se amplió al sureste de la cuenca precisamente junto al margen del curso principal del río, para finalmente en el año 2014 ampliarse significativamente a la zona norte de la cuenca donde se encuentran la mayor parte de los tributarios del río y la altitud alcanza los $1.000 \mathrm{msnm}$. Esta localización presenta buena calidad de los suelos: muy profundos, porosos y ricos en materia orgánica.

En relación con el cacao nacional fino de aroma, para 1990 la cuenca del río Vinces presentaba importantes superficies cubiertas cuyas plantaciones mayormente se encontraban al sur de la subcuenca y en poca superficie en el sector norte de la cuenca. Durante la década del 2000, las plantaciones de cacao nacional fino de aroma dejaron de ser productivas y prácticamente fueron abandonadas para posteriormente ser reemplazadas por el clon de cacao CCN51 que es el que en la actualidad se ha extendido desde el sur, centro y norte de la cuenca del río Vinces gracias a su precocidad productiva e intensidad de producción sobre todo en verano.

La palma aceitera conocida también como palma africana, para el año 1990 tenía una modesta presencia en la zona noroccidental de la cuenca en terrenos con una cota que llegaba a los 600 msnm. Para el año 2014 se expandió desde el sur, centro y norte de la cuenca en terrenos con distancias cercanas a cursos de agua superficial menores, pero sin llegar a la expansión que ha tenido los cultivos de banano y cacao.

\section{Demanda de agua para riego}

En relación con el volumen de agua para riego de cultivos agrícolas, para la cuenca del río Vinces, según CISPDR

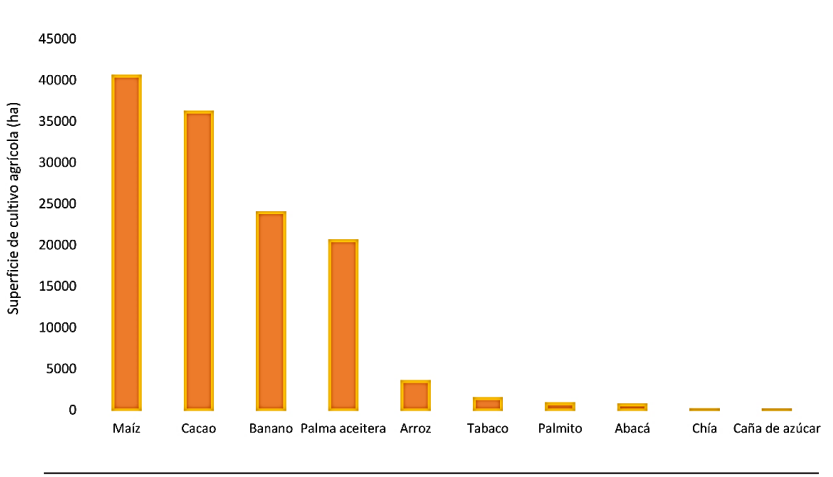

Figura 5. Distribución de cultivos agrícolas (127.345,70 ha) en cuenca del río Vinces (Ecuador) (MAGAP, 2014). Fuente: Elaboración propia.

(2016), corresponde a 573,06 hm3 (figura 4). Este volumen fue distribuido de acuerdo a los cultivos agrícolas existentes en la cuenca del río Vinces (figura 5).

La Secretaría del Agua (SENAGUA) ha otorgado concesiones de agua para riego en el período comprendido entre los años 1980 - 2018 para la cuenca del río Vinces de acuerdo a lo indicado en la figura 6 .

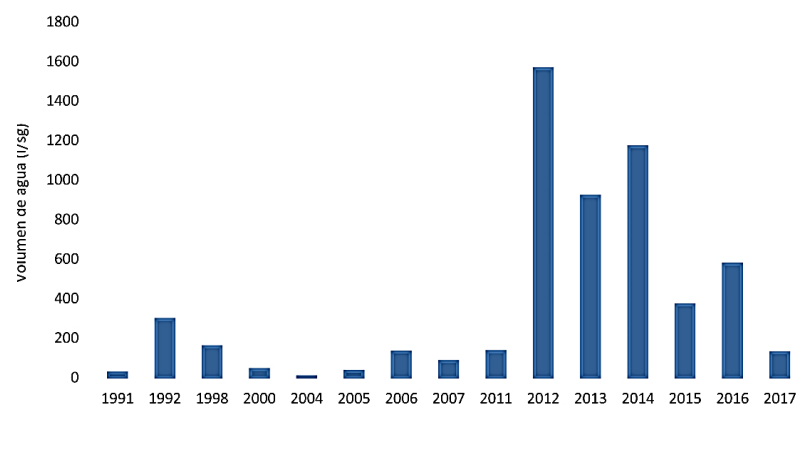

Figura 6. Concesiones de agua (I/sg) para riego de cultivos agrícolas en la cuenca del río Vinces (Ecuador) para el período 1980-2018. Fuente: elaboración propia.

En la figura 6 se observa una importante variación interanual de las concesiones de agua por parte de la Secretaría del Agua, debido a que en el período comprendido entre los años 1991 y 2011 no hubo mayor control para los usuarios agrícolas por la dificultad que le significaba esta labor a los órganos de control de carácter centralizado, propiciándose que de manera clandestina se abusara del recurso hídrico para el riego agrícola. A partir del 2008, que por Decreto ejecutivo 1088 se creó la Secretaria Nacional del Agua (SENAGUA), esta situación cambió gracias a la puesta en funcionamiento de las oficinas descentralizadas de atención a la ciudadanía, como es el caso de la oficina 
de atención al cliente de Quevedo, la misma que inició con un proceso de emisión y control de concesiones de agua para riego de manera equitativa en la cuenca del río Vinces de acuerdo a la extensión de superficie de terrenos de los productores agrícolas (SENAGUA, 2011).

Los recursos hídricos de Ecuador son abundantes. El volumen de agua que proveen todos los sistemas hidrográficos en el territorio nacional llega a los $432.000 \mathrm{hm}^{3}$ en la estación lluviosa, mientras que en la época seca alcanza tan solo los $146.000 \mathrm{hm}^{3}$, de los cuales $115.000 \mathrm{hm}^{3}$ corresponden a la vertiente del Pacífico y $317.000 \mathrm{hm}^{3}$ a la Amazónica. No obstante, de ello, la disponibilidad general para el país es de solo el $34 \%$, es decir $146.000 \mathrm{hm}^{3}$, esta fluctuación de disponibilidad se debe a la irregular distribución espacial y temporal de las precipitaciones dado que en los ocho meses de verano la precipitación se reduce en relación al invierno en un 90\% según Galárraga- Sánchez (2000) citado por MAGAP Ecuador (2013).

Las precipitaciones en el país se distribuyen en los períodos de invierno y verano, de acuerdo a datos recogidos durante treinta y cinco años en la estación meteorológica Pichilingue del Instituto Nacional Autónomo de Investigaciones Agropecuarias (INIAP PICHILINGUE) ubicada en la parte central de la cuenca del río Vinces. El promedio mensual de las lluvias en el período invernal de enero a abril es de $423,78 \mathrm{~mm}$, mientras que en la época de verano de mayo a diciembre la media es de $67,70 \mathrm{~mm}$, por lo que resulta imperioso el regadío de los cultivos agrícolas en la cuenca en el largo período de verano (Caicedo, Cadena, Alcívar, Veloz \& Montecé, 2016).

De acuerdo a comunicaciones de informantes clave dedicados a la producción de bananos, una hectárea de cultivo de banano en producción requiere ser regada por aspersión subfoliar tres veces por semanas durante dos horas en cada ocasión. Los aspersores subfoliares alcanzan un caudal de $680,21 \mathrm{~L} /$ hora siendo el espaciamiento entre ellos de $12 \mathrm{~m}$ x $14 \mathrm{~m}$, con eficiencia de aplicación de 90 $\%$ (Caicedo et al., 2016).

La producción de banano de exportación en la cuenca del río Vinces está asociada a un uso no optimizado del recurso agua para riego. El cultivo de banano requiere grandes cantidades de agua, el estudio realizado en el centro de la cuenca del río Vinces determinó que una planta consume de manera aproximada 30 litros de agua diaria en días soleados, 24 litros en días seminublados y 12,5 litros en días nublados, esto puede ser necesario especialmente en época seca siendo aplicado por gravedad, aspersión, inundación o goteo y es precisamente en esta última parte que la carencia de los estudios técnicos del sitio derivan en la elección de un método de riego que genera tasas elevadas de desperdicio de agua (Rodríguez, 2009).

En la cuenca del río Vinces, el cacao en producción que existe corresponde al clon CCN-51 que no tolera la escasez de agua, pero a diferencia del banano no tolera el encharcamiento. De acuerdo a productores de cacao CCN-51, una hectárea de cacao debe ser regada una vez por semana por el lapso de dos horas y es en los meses de verano cuando el riego es imperioso dado que es en esta época en que el cacao CCN-51 tiene su mayor productividad. La precipitación óptima para el cacao $\mathrm{CCN}-51$ es de 1.600 a $2.500 \mathrm{~mm}$ distribuidos durante todo el año (Paredes, 2004). Los requerimientos de agua en el cultivo de palma aceitera no son constantes ni únicos, por lo que para calcularlos es necesario considerar la capacidad de almacenamiento del suelo en la zona de las raíces, la disponibilidad de agua al alcance de las palmas y los requerimientos de agua para procesos fisiológicos y físicos (Granada, 2001).

Las plantaciones de palma aceitera en la cuenca del río Vinces no cuentan, en su mayor parte, con sistema de riego debido a que se desarrollan en suelos moderadamente húmedos. Sin embargo, existe una importante discusión en torno al hecho de que las plantaciones intensivas de palma aceitera reducen significativamente los niveles de los acuíferos y vertientes circundantes. El cultivo de palma aceitera presenta su óptimo desempeño en regiones con una precipitación menor a $2.000 \mathrm{~mm}$ al año con una buena distribución, los valores medios mensuales en relación con buenos rendimientos son superiores a los $150 \mathrm{~mm}$. No obstante, la palma aceitera posee de manera natural gran capacidad para sobrevivir a períodos prolongados de sequía gracias a características morfológicas y fisiológicas, además de la habilidad de abortar las inflorescencias, la variación estacional en los picos de producción de racimos y la movilización de reservas que le permiten compensar la menor tasa de fotosíntesis debido al cierre de estomas (Nouy, Baudouin, Djegui \& Omore, 1999). En un estudio realizado sobre tres híbridos variedad vegetativa tenera de palma aceitera, una de las más sembrada en Ecuador por su resistencia a plagas y en enfermedades, se reportó un efecto positivo del riego en el comportamiento de cada uno de los híbridos en estudio para las variables altura de planta, relación sexo y Producción (Reinoso, 2008).

\section{Discusión}

El análisis temporo-espacial de la cobertura agrícola de la cuenca del río Vinces en las últimas tres décadas permitió 
visibilizar el crecimiento sostenido de la producción agrícola a través del desarrollo de monocultivos intensivos de exportación. Como afirman Galicia, Corona, PalacioPrieto, Bürgi \& Hersperger (2016), la topografía con sus rasgos de pendiente y altitud está relacionada con la distribución del uso y la cobertura del suelo, mientras que las variables socioeconómicas más importantes que lo afectan son las distancias de caminos, áreas rurales, centros urbanos y centros turísticos. No obstante, los factores físicos mencionados no fueron una limitante en el caso de la cuenca del río Vinces, donde la expansión de los monocultivos de agroexportación fue hacia zonas de gran altitud e importante gradiente del terreno y con un impacto en la demanda de agua superficial para riego en los meses de verano.

La presencia de monocultivos exóticos en la cuenca del río Vinces ha cambiado significativamente el paisaje de una de las áreas más ricas en recursos naturales de Ecuador. A la fecha existen muy pocos remanentes de parches boscosos en la parte media y baja de la cuenca resultando infructuosos los controles que ejerce la autoridad ambiental competente, en esta problemática coinciden (Lambin \& Turner, 2001) quienes señalan que la intervención humana es la que ha provocado mayores transformaciones sobre la superficie terrestre, pese a que las modificaciones ambientales pueden ocurrir de manera natural o pueden ser del tipo antropogénicos siendo los resultados generales cambios de cobertura vegetal y uso del suelo que traen consigo efectos que empobrecen el potencial biológico y cultural. Pacheco et. al (2018) aplicaron el índice de Shannon a provincias de interés en Ecuador para 2014. Los resultados dan cuenta que las provincias con mayor diversificación agrícola son Imbabura, El Oro, Tungurahua, Cotopaxi, Loja y Cañar, mientras que las provincias con menor diversificación son Morona Santiago, Pastaza, Zamora Chinchipe y finalmente Santa Elena. Los resultados de este análisis excluyen a la provincia de Los Ríos, área eminentemente agrícola que conforma la cuenca del río Vinces.

El monocultivo intensivo de banano se ha expandido principalmente por los grupos económicos de poder cuyos lotes superan las 100 hectáreas, lo que genera una presión muy alta por el recurso hídrico de la cuenca para el riego. Frecuentemente se puede observar que no respetan los caudales concesionados por la autoridad ambiental y en muchos casos han sido sancionados económicamente, pero pagan sus multas y siguen a provechando clandestinamente el agua para riego del río Vinces. Esta realidad vivida en la cuenca no es muy diferente a lo que ocurre en el área rural de Bogotá, donde la ampliación de la frontera agrícola ha llevado a la desaparición casi total de las áreas de amortiguación del páramo, esto se relaciona con las formas de aprovechamiento económico del suelo, debido a varios latifundios que han venido siendo arrendados a terceros, precipitando el deterioro ecosistémico e hídrico de la cuenca (Hernández, Rojas \& Sánchez, 2013).

En la práctica, los productores bananeros del Ecuador desconocen los volúmenes de agua que utilizan mediante el riego por aspersión, ya que se asume que siempre se irriga hasta que el suelo se encuentra saturado (Zarate \& Kuiper, 2013) pese a que lo ideal sería $27.500 \mathrm{~m}^{3}$ de agua para riego por ha/año. Los productores ecuatorianos señalan además que en las cuencas donde se produce banano existe una competencia por el recurso hídrico, notándose la disminución en la disponibilidad de agua en época seca especialmente, esto ha provocado cambios en la hidrología de los ríos por factores como la deforestación y uso del suelo inapropiado. De acuerdo con Zarate \& Kuiper (2013), la situación en Perú no es distinta ya que una hectárea anual de banano en producción requiere $28.500 \mathrm{~m}^{3}$ de agua para riego y ante las precipitaciones mínimas existen proyecciones de aumento del estrés hídrico en los años venideros, ya que en la actualidad no se respetan los caudales mínimos ambientales, con repercusiones graves para los ecosistemas.

Los resultados del estudio temporo-espacial de la cobertura agrícola de la cuenca del río Vinces durante el período 1990-2014 permitió identificar las tendencias recientes en los patrones de la ocupación del suelo. Morales, Carrillo, Farfán \& Cornejo (2016) indican que el análisis espacialcuantitativo sobre los cambios en la cobertura vegetal y el uso del suelo entre 1979-2013 en la región de Bahía de Banderas, México, generó información valiosa para el monitoreo de los recursos naturales con implicaciones en el ciclo hidrológico, la biodiversidad, la erosión del suelo y el clima local, entre otros aspectos relevantes. Asimismo, Florez-Yepes, Rincon-Santamaría, Cardona \& Alzate-Alvarez (2017) manifiestan que la aplicación del análisis multitemporal permite determinar los cambios e impactos ambientales más significativos a través del tiempo permitiendo conocer las interrelaciones entre los elementos que lo componen y las actividades antrópicas.

\section{Conclusiones}

Entre los principales monocultivos agrícolas existentes en la cuenca del río Vinces se encuentran el banano, el cacao y la palma aceitera. En relación con el cambio de uso del suelo, se observó que, para el caso del banano, uno de los principales cultivos de exportación de Ecuador, en el 
período presentado, muestra una expansión del $212 \%$. En el caso del monocultivo de cacao muestra una expansión del $329 \%$. Por otro lado, el monocultivo de palma aceitera o palma africana presenta una ligera disminución (del 4\%). En relación con la expansión de la frontera agrícola de estos tres monocultivos de agroexportación, el cultivo de banano se expandió de una manera regular en el centro y noreste de la cuenca del río Vinces, mientras que el cultivo de cacao lo hizo de manera regular en el centro, noreste y sur de la cuenca, mientras que el cultivo de palma aceitera tuvo un patrón de expansión regular en el centro, norte y sur de la cuenca y de manera irregular en el sector noroccidental de la cuenca.

Destaca el cultivo de banano por ser el que más presión ejerce sobre el recurso hídrico por el alto volumen de riego que requiere para mantener sus niveles de productividad (durante la época seca consume aproximadamente entre 30 litros de agua diaria en días soleados y 12,5 litros en días nublados). La aplicación de agua de riego es una necesidad imperiosa para obtener rendimientos altos y estables de este cultivo, la principal fuente de agua son los cursos hídricos superficiales. En relación con esta producción, es importante destacar que los recorridos en campo permitieron observar conexiones clandestinas al eje hídrico principal de la cuenca, así como a la de sus afluentes, lo que evidencia la falta de recurso económico y humano para controlar la amplia área de la cuenca.

Si bien existe una regulación de la ampliación de la siembra de cultivo de banano en la cuenca del río Vinces con un único objetivo económico que es frenar la sobreproducción de la fruta para mantener su precio, esta no se cumple efectivamente. No existe un contexto de protección ambiental, por lo que el panorama para el río Vinces en un mediano plazo podría ser el de prácticamente de sequía total.

De cumplirse las tendencias de cambio en el patrón de precipitaciones para Ecuador en el contexto del cambio climático, la cuenca del río Vinces sufriría consecuencias negativas asociadas al déficit hídrico. Podrían quedar improductivas importantes áreas agrícolas de secano cubiertas por monocultivos agrícolas destinados mayormente a la agroexportación que exigen para su productividad importantes volúmenes de agua de irrigación captados mayormente de los cursos superficiales de la cuenca, para de esta manera lograr altos niveles de producción.

\section{Referencias}

Borbor-Cordova, M. J., Boyer, E. W., McDowell, W. H., \& Hall, C.A. (2006). Nitrogen and phosphorus budgets for a tropical watershed impacted by agricultural land use: Guayas, Ecuador. Biogeochemistry, 79(1-2), 135-161. https://doi.org/10.1007/s10533-006-9009-7

Cadilhac, L., Torres, R., Calles, J., Vanacker, V. \& Calderón, E. (2017). Desafíos para la investigación sobre el cambio climático en Ecuador. Neotropical Biodiversity, 3(1), 168-181. https://doi.org/10.1080/ 23766808.2017.1328247

Caicedo, C., Cadena, D., Alcívar T., Veloz, A. \& Montecé, F. (2016). Análisis del comportamiento de las precipitaciones en Quevedo - Ecuador, para la planificación de cultivos. European Scientific Journal, 12(33), 212-220. http://dx.doi.org/10.19044/ esj.2016.v12n33p212

Chacón, V. (24 de octubre de 2012). Denuncian contradicciones en cifras sobre tierras dedicadas a la piña. Semanario Universidad de la UCR. Recuperado de https://historico.semanariouniversidad.com/pais/ denuncian-contradicciones-en-cifras-sobre-tierrasdedicadas-a-la-pia/

CISPDR. (2014). Planificación Hídrica Nacional del Ecuador (2014-2035). Phase II Report.

CISPDR. (2015). Plan Hidraúlico Regional de la Demarcación Hirográfica Guayas. Memoria y Anexos.

CISPDR (2016). Plan Hidráulico Regional de la Demarcación Hidrográfica Guayas.

Damanik-Ambarita, M. N., Boetsa, P., Nguyen, H. T., Eurie, M. A., Everaerta, G., Locka, K., Sasha, P. L., Suharevae, N., Bennetsena, E., Gobeyna, S., LongHo, T., Dominguez-Granda \& Goethalsa, P. L. (2018). Impact assessment of local land use on ecological water quality of the Guayas river basin (Ecuador). Ecological Informatics, 48, 226 - 237. https://doi. org/10.1016/j.ecoinf.2018.08.009

Damiani, O. (2001). Diversificación de la agricultura y reducción de la pobreza rural: cómo los pequeños 
agricultores y asalariados rurales son afectados por la introducción de cultivos no-tradicionales de alto valor en el noreste de Brasil. Inter-American Development Bank. Recuperado de https://publications.iadb.org/es/ publicacion/14529/diversificacion-de-la-agriculturay-reduccion-de-la-pobreza-rural-como-los

Espinosa, J. \& Rivera, D. (2016). Variations in water resources availability at the Ecuadorian páramo due to land-use changes. Environ Earth Sci, 75(1173), 1-15. https://doi.org/10.1007/s12665-016-5962-1

Florez-Yepes, G., Rincon-Santamaría, A., Cardona, P., \& Alzate-Alvarez, A. (2017). Análisis multitemporal de las coberturas vegetales en el área de influencia de las minas de oro ubicadas en la parte alta del sector de Maltería en Manizales, Colombia. DYNA, 84(201), 95-101. https://doi.org/10.15446/dyna. v84n201.55759

Frappart, F., Bourrel, L., Brodu, N., Riofrío Salazar, X., Baup, F., Darrozes, J. \& Pombosa, R. (2017). Monitoring of the Spatio-Temporal Dynamics of the Floods in the Guayas Watershed (Ecuadorian Pacific Coast) Using Global Monitoring ENVISAT ASAR Images and Rainfall Data. Water, 9(1), 1-20. https:// doi.org/10.3390/w9010012

Galicia, L., Corona, R., Palacio-Prieto, J., Bürgi, M. \& Hersperger, A. (2016). Local deforestation patterns and their driving forces of tropical dry forest in two municipalities in Southern Oaxaca, Mexico (19852006). Investigaciones Geográficas, 91, 86-104. http://dx.doi.org/10.14350/rig.50918

Gaybor, A., Ramos, A., Tamayo, C., Isch, E. \& Arroyo, A. (2008). El despojo del agua y la necesidad de una transformación urgente. Quito: Foro de los recursos hídricos.

Gregory, K. J., Gurnell, A. M., \& Petts, G. E. (2002). Restructuring physical geography. Transactions of the Institute of British Geographers, 27(2), 136-154. https://doi.org/10.1111/1475-5661.00046

Granada, J. (2001). Riego en palma de aceite. En Actualización en la producción de palma aceitera (pp. 219-224). Maturín (Venezuela).

Goletti, F. (1999). Agricultural Diversification and Rural Industrialization as a Strategy for Rural Income Growth and Poverty Reduction in Indochina and Myanmar. Recuperado de http://cdm15738. contentdm.oclc.org/utils/getfile/collection/ p15738coll2/id/125722/filename/125753.pdf

Gudynas, E. (2013). Extracciones, extractivismos y extrahecciones: Un marco conceptual sobre la apropiación de recursos naturales. Observatorio del Desarrollo, CLAES, 18, pp. 1-18. Recuperado de http://ambiental.net/2013/03/definicionesextracciones-extractivismos-extrahecciones/

Hernández, A., Rojas, R., Sánchez, F. (2013). Cambios en el uso del suelo asociados a la expansión urbana y la planeación en el corregimiento de Pasquilla, zona rural de Bogotá (Colombia). Cuadernos de Geografia, 22(2), 257-271. https://doi.org/10.15446/ redg.v22n2.37024

INOCAR. (2010). Memoria Técnica de la comisión realizada en el área del Río Guayas sur. Guayaquil: Instituto Oceanográfico de la Armada.

Keating, M. (1993). The Earth Summits agenda for change: A plain language version of Agenda 21 and the Other Rio Agreements. Geneva (Switzerland): Centre for Our Common Future.

Knauer, K., Gessner, U., Fensholt, R., Forkuor, G. \& Kuenzer, C. (2017). Monitoring agricultural expansion in Burkina Faso over 14 years with 30 $\mathrm{m}$ resolution time series: The role of population growth and implications for the environment. Remote Sensing, 9(2), 132. https://doi.org/10.3390/rs9020132

Lambin, E.F. \& B. Turner. (2001). The causes of landuse- cover change. Global Enviromental Change, (11)4, 261-269. https://doi.org/10.1016/S09593780(01)00007-3

Lambin, E. F. \& Mayfroidt P. (2011). Global land use change, economic globalization, and the looming land scarcity. Proceedings of the National Academy of Sciences of the Unites States of America (PNAS), 108(9), 3465-3472. https://doi.org/10.1073/ pnas. 1100480108

MAGAP. (2012). Ecuador preside Comité Económico de la Organización Internacional del Cacao. Recuperado de https:/www.agricultura.gob.ec/ ecuador-asume-presidencia-de-la-organizacionmundial-del-cacao/ 
MAGAP. (2013). Plan Nacional de Riego y Drenaje 2012 - 2027. Recuperado de http://es.scribd.com/ doc/127451977/Libro-Plan-Nacional-de-Riego-yDrenaje

MAGAP. (2014). Land Use and Lan Cover Map. Years 2014-2015, 1:25,000 Scale. Recuperado de http:/ ide.sigtierras.gob.ec/geoportal/

MAGAP. (2015). Land Use and Lan Cover Map. Years 2014-2015., 1:25,000 Scale. Recuperado de http:/ ide.sigtierras.gob.ec/geoportal/

Morales, J., Carrillo, F., Farfán, L., Cornejo, L. (2016). Cambio de cobertura vegetal en la región de bahía de banderas, México. Revista colombiana de Biotecnología, 18(1), 7-16. Recuperado de https:// revistas.unal.edu.co/index.php/biotecnologia/index

Nené-Preciado, A., González, G., Mendoza, M. \& Silva, F. (2017). Cambio de cobertura y uso de suelo en cuencas tropicales costeras del Pacífico central mexicano. Investigaciones Geográficas, 94, 64-81. https://doi.org/10.14350/rig.56770

Nouy, B., Baudouin L., Djegui N. \& Omore A. (1999). Le palmier ahuile en conditions hydriques limitantes. Plantations, recherche, developpmement, 6(1), 3145. Recuperado de http://agritrop.cirad.fr/391747/1/ document_391747.pdf

Pacheco, J., Ochoa-Moreno, W. S., Ordoñez, J. \& Izquierdo-Montoya, L. (2018). Agricultural diversification and economic growth in Ecuador. Sustainability, 10(7), 2257. https://doi.org/10.3390/ su10072257

Pan, W. K., Walsh, S. J., Blisborrow, R. E., Frizzelle, B. G., Erlien, C. M., \& Baquero, F. (2004). Farm-level models of spatial patterns of land use and land cover dynamics in the Ecuadorian Amazon. Agriculture Ecosystem and Environment, 101(2-3), 117-134. https://doi.org/10.1016/j.agee.2003.09.022

Paredes, M. (2004). Manual del cultivo del cacao. Programa para el desarrollo de la Amazonía, Proamazonía. Perú: Ministerio de Agricultura. Recuperado de http://canacacao.org/biblioteca/

Ramankutty, N., Heller, E. \& Rhemtulla, J. (2010). Prevailing myths about agricultural abandonment and forest regrowth in the United States. Annals of the
Association of American Geographers, 100(3), 502512. https://doi.org/10.1080/00045601003788876

Raymond, K. L. \& Vondracek, B. (2011). Relationships among rotational and conventional grazing systems, stream channels, and macroinvertebrates. Hydrobiologia, 669, 105-117.https://doi.org/10.1007/ s10750-011-0653-0

Reinoso V. (2008). Influencia del riego en el comportamiento de tres hibridos tenera de palma aceitera (Elaeis guineensis Jacq) de diferentes orígenes (4to año de ejecución) [tesis de grado, Universidad Central del Ecuador].

Rindfuss, R. R., Entwisle, B., Walsh, S. J., Mena, C. F., Erlien, C. M. \& Gray, C. L. (2007). Frontier land use change: synthesis, challenges, and next steps. Annals of the Association of American Geographers, 97(4), 739-754. https://doi.org/10.1111/j.14678306.2007.00580.x

Rodríguez C. (2009). Estudio de Factibilidad para la producción y comercialización de banano (Musa sp.), variedad gran enano Cavendish, en Quevedo, provincia de Los Ríos [tesis de grado, Universidad San Francisco de Quito].

Salmoral, G., Khatun, K., Llive, F. \& Madrid, C. (2018). Agricultural development in Ecuador: A compromise between water and food security? Journal of Cleaner Production, 202(20), 779-791. https://doi. org/10.1016/j.jclepro.2018.07.308

SENAGUA. (2009). Unidades Hidrográficas (GIS). Recuperado de https://aplicaciones.senagua.gob. ec/servicios/descargas/archivos/delimitacioncodificacion-Ecuador.pdf

SENAGUA. (2011). Informe de gestión 2008-2010. Una gestión diferente de los recursos hídricos. Ecuador.

Seoane, J. (2013). Modelo extractivo y acumulación por despojo. En Seoane, J.; Taddei, E. y Algranati, C. (Eds.), Extractivismo, despojo y crisis climática (pp. 21-41). Buenos Aires: Editorial El Colectivo.

SEPSA (Secretaría Ejecutiva de Planificación Sectorial Agropecuaria). (2001). Boletín Estadístico Agropecuario Nro.12. Recuperado de http://www. mag.go.cr/biblioteca_virtual/bibliotecavirtual/ a00029.PDF 
SEPSA (Secretaría Ejecutiva de Planificación Sectorial Agropecuaria). (2013). Boletín Estadístico Agropecuario Nro. 23, serie cronológica 2009-2012. Recuperado de http://www.mag.go.cr/biblioteca virtual/bibliotecavirtual/a00322.pdf

Silvetti, F., Soto, G., Cáceres, D. \& Cabrol, D. (2013). ¿Por qué la legislación no protege los bosques nativos de Argentina? Conflictos socioambientales y políticas públicas. Mundo Agrario, 13(26). Recuperado en https://ri.conicet.gov.ar/handle/11336/794

Svampa, M. (2008). Cambio de época. Movimientos sociales y poder político. Buenos Aires: Siglo XXI/ CLACSO.

World Bank. (2018). Population, Total - Ecuador. World Bank Development Data Group. Recuperado de https://data.worldbank.org/indicator/ SP.POP.TOTL? end $=2016 \&$ locations $=E C \&$ start $=1960 \&$ view $=$ chart

Yacoub, C., Duarte B. \& Boelens, R. (Eds.). (2015). Agua y ecología política: el extractivismo en la agroexportación, la minería y las hidroeléctricas en Latinoamérica (serie Agua y Sociedad, sección Justicia Hídrica, 22). Quito: Abya-Yala / Justicia Hídrica.

Zarate, E. \& Kuiper. D. (2013). Evaluación de Huella Hídrica de la banana para pequeños productores en Perú y Ecuador. Switzerland: Good Stuff International. Recuperado de http://www.goodstuffinternational. com/images/PDF/Reporte\%20GSI HH Bananas pequenos_productores.pdf

Zeolla, N. H. (2013). Costos y rentabilidad del cultivo de soja en Argentina. Informe Económico Especial, II CELSO (julio). Recuperado de https://www.ceso. com.ar/sites/www.ceso.com.ar/files/ceso_sector agropecuario_3.pdf

Zimmerer, K., S. (2010). Retrospective on nature-society geography: Tracing trajectories (1911-2010) and reflecting on translations. Annals of the Association of American Geographers, 100(5), 1076-1094. https:// doi.org/10.1080/00045608.2010.523343

\section{Agradecimientos}

Departamento de Geografía y Turismo de la UNS y a la Facultad de Ciencias Ambientales de la UTEQ, a la SENAGUA y al MAGAP del Ecuador. 